\title{
Comparative Study of Image Reconstruction Based on Compressive Sensing
}

\author{
Himani A. Shah*1, Mr. Dipak Agrawal*2, Mr. Nimit Modi*3, Dr. Sheshang Degadwala*4 \\ ${ }^{*} 1$ M.E. Student of Computer Department, GTU/ Sigma Institute of Engineering, Vadodara, Gujarat, India \\ *2 Ass. Prof .of Computer Department, GTU/ Sigma Institute of Engineering, Vadodara, Gujarat, India \\ *3 Ass. Prof. of Computer Department, GTU/ Sigma Institute of Engineering, Vadodara, Gujarat, India \\ *4 Head of Computer Department, GTU/ Sigma Institute of Engineering, Vadodara, Gujarat, India
}

\begin{abstract}
Compressive sensing based image reconstruction that improves the algorithm to applying different approach which is DWT and DCT. First, by using wavelet transform, wavelet low frequency of the sub bands in which the image is decomposed in to low frequency and high frequency wavelet coefficients, second is to applied DCT on low frequency coordinates and construct the different transformation matrix. Use the measurement matrix measure the high frequency coefficient components and combine with DCT low frequency components image and sparse signal output is applied on compressive sensing. In compressive sensing, random measurement matrices are generally used and $\ell 1$ minimisation algorithms often use linear programming to cover sparse signal vectors. But explicitly constructible measurement matrices providing performance guarantees were and Ł1minimisation algorithms are often demanding in computational complexity for applications involving very large problem dimensions. To improve the PSNR (pick signal to noise ratio) of reconstructions image uses different coding such as Huffman and Arithmetic.
\end{abstract}

Keywords : Compressive Sensing; DWT; DCT; Huffman; Arithmetic.

\section{INTRODUCTION}

Compressive sensing is reconstruction of the signals or images that contains common type of structures that, enables and intelligent and concise representation [2]. Image is compressed by partial numerical of projections, hence it ashen the saddle with processing, storage, and transmission. At the receiver, the sparse recovery surety to recovered images with less differences conflicts with the base truth [5]. To reduce the size the data in terms as a bit rate of the file without any degradation or discarded any important details of image or signal and it would be affected to reduce transmission time and also storage purpose.

In digital multimedia technology is applicant in almost every field of the communication such as in digital images and video processing uses increased tremendously because of the advance computers and digital cameras [6]. Therefore, the size of the data could be affected on memory space, bandwidth, and transmission time and transmission rate. So, compression method is applied to reduce the size of the data in terms of the redundancy and discarded that all components therefore less time will be required to download these images. Best performance 
of the image compression techniques is wild area in digital communication [7].

There are several image compression techniques but mainly applicant on images such as Discrete Cosine Transform (DCT), Discrete Wavelet Transform (DWT), and Huffman Technique, arithmetic coding etc. At last reconstruction the image to use linear programming to cover sparse signal vectors techniques and improve compressive sensing algorithm.

In compressive sensing the information acquisition process is linear; the problem reduces to solving a linear system of equations. In mathematical terms, the observed data $\mathrm{y} \in \mathrm{Cm}$ is connected to the signal $\mathrm{x} \in$ $\mathrm{CN}$ of interest via $\mathrm{Ax}=\mathrm{y}$. The matrix $\mathrm{A} \in \mathrm{Cm} \times \mathrm{N}$ models the linear measurement (information) process. Then one tries to recover the vector $\mathrm{x} \in \mathrm{CN}$ by solving the above linear system. Traditional wisdom suggests that the number $\mathrm{m}$ of measurements, i.e., the amount of measured data, must be at least as large as the signal length $\mathrm{N}$ (the number of components of $\mathrm{x}$ ) [11].

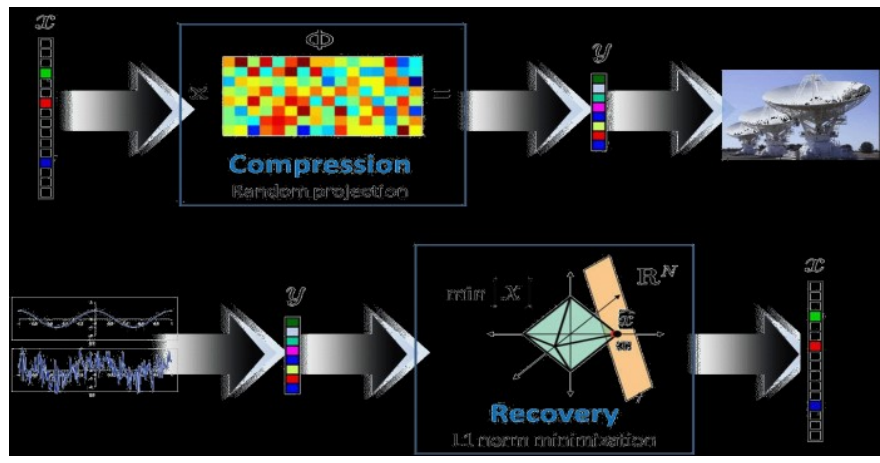

Figure 1: Basic System Blocks Compressive Sensing

\section{RELATED WORKS}

In this paper [1] Jian Chenl; Yatin Gao describes improve the CS image reconstruction performance by utilizing the structure redundant information. The proposed MRCS in the paper combines the sparsity of an image on various transform domains corresponding to the different characteristics of image structure.
In this paper [2] Xiumei Li , Guoan Bi describes image reconstruction based on the improved Bayesian compressive sensing using the single-level wavelet transform. By using the single-level wavelet transform, the image is decomposed into low-frequency and high-frequency wavelet coefficients.

In this paper [3] Muhammad Ali Qureshi, M. Deriche discussed the algorithm starts with a traditional multilevel 2-DWavelet decomposition, which provides a compact representation of image pixels. Then they introduce a new approach for rearranging the wavelet coefficients into a structured manner to formulate sparse vectors.

In this paper [4] Xiumei Li, Guoan Bi discussed the image reconstructions using the improved compressive sensing algorithm based on the wavelet transform. First, by using the wavelet transform, the image is decomposed into low-frequency wavelet coefficients and high-frequency wavelet coefficients; second, keep the low frequency wavelet coefficients unchanged, use the measurement matrix to measure the high-frequency wavelet coefficients, and then combine with the unchanged low-frequency coefficients to reconstruct the image.

In this paper [5] Narendra N, M Girish Chandra and B S Adiga describes Compressive Sensing (CS) provides a solid theoretical framework for sparse signal recovery. In this work, they concentrate on recovering the foreground object which can be represented as a sparse vector using wavelets. The method revolves around the CS framework by judiciously using the complex field $\mathrm{BCH}$ codes and the syndrome as measurements to achieve our goal of robust background subtraction using reduced number of measurements. 


\section{METHODOLOGY}

\section{A. Transformation Techniques}

I. Discrete Wavelet Transform (DWT) $[3,5]$ : Discrete Wavelet Transform (DWT) Wavelets are small parts of the large wane of the signal which can be define mathematically to differentiate its coordinate of the system and represented the signal in to the different domain which is more suitable for compression and also catch further information. Wavelet transform is multi resolution inherent filter coding, more robust for the transmission and decoding errors. Wavelets are the model based structure to decomposing signals or images, and increasing resolutions so resultant more resolution structure gets the more detailed or information of the image. Wavelet is located coefficients, from the zero and increases and then decreases and then waves that drop to zero.

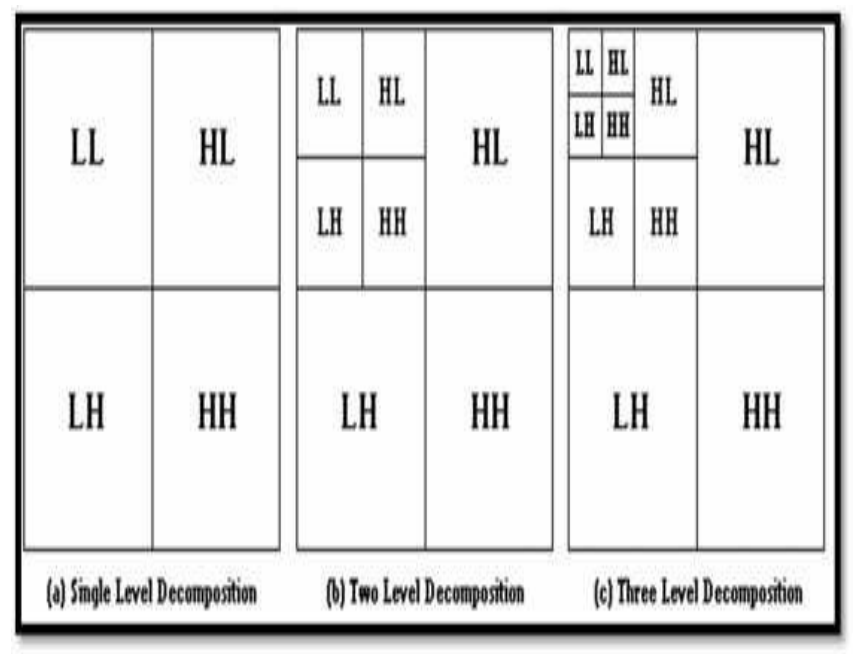

Figure 2: Pyramidal Decomposition of DWT

II. Discrete Cosine Transforms (DCT) $[8,10]$ : DCT (discrete cosine transforms) is developed in 1974 which is great achievement for the research group working on image compression. The DCT refers as a discrete-time distribution of the Fourier-cosine series. It is some close to DFT, a technique for converting a single into elementary frequency components. Thus DCT can be computed with a Fast Fourier Transform (FFT) like algorithm in $\mathrm{O}(\mathrm{n} \log \mathrm{n})$ operations. DCT is revalued and provides accuracy then DFT of a single with local area and details by few coefficients. The
DCT of a discrete signal $\mathrm{x}(\mathrm{n}), \mathrm{n}=0,1, \ldots, \mathrm{N}-1$ is defined for $1 \mathrm{D}$ and $2 \mathrm{D}$ analysis and synthesis process equations. There are main step is to dividing image in to the small blocks applied DCT on each block of the pixel. DCT gets higher energy compaction with applied fewer coefficient. After that applied quantization in each component and set the bit allocation.

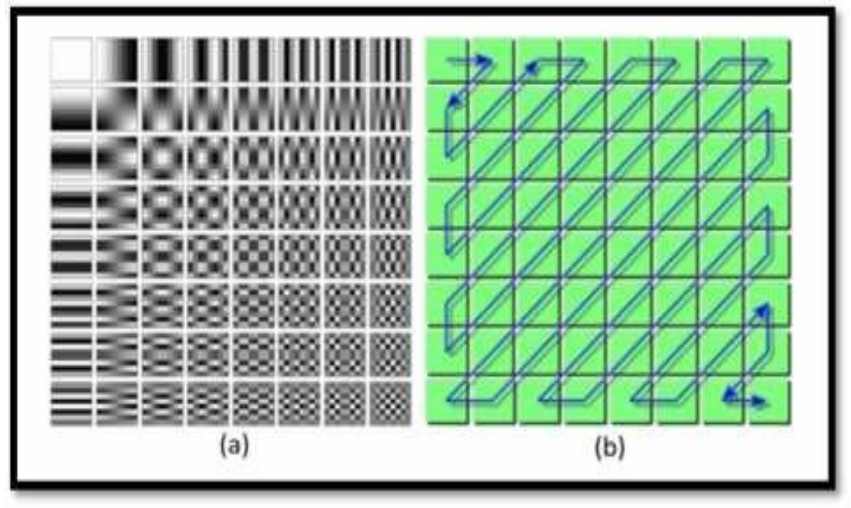

Figure 3: (a) Example DCT patterns with resolution of 8 X 8; (b) The order of DCT Patterns

\section{B. Encoding Techniques:}

I. Arithmetic [13]: The sparse measurements taken by the technique of compressed sensing are to be encoded by arithmetic encoding. Arithmetic coding is a form of entropy coding used in lossless data compression. When a string is arithmetic encoded, frequently used characters will be stored with fewer bits and not-so-frequently occurring characters will be stored with more bits, resulting in fewer bits used in total. Arithmetic coding differs from other forms of entropy encoding such as Huffman coding in that rather than separating the input into component symbols and replacing each with a code, arithmetic coding encodes the entire message into a single number, usually a fraction $n$ where $(0.0 \leq n<1.0)$, called a Tag value. The encoder divides the interval into sub-intervals, each representing a fraction of the current interval proportional to the probability of that symbol in the current context. Whichever interval corresponds to the actual symbol that is next to be encoded becomes the interval used in the next step. When all symbols have been encoded, the resulting interval unambiguously identifies the sequence of 
symbols that produced it. If the same final interval and model that is being used are available, the symbol sequence that must have entered the encoder to result in that final interval can be reconstructed. It is not necessary to transmit the final interval, however; it is only necessary to transmit one fraction that lies within that interval.

B. Huffman [11]: The main objective of CS based quantized prediction Huffman encoder is to reduce the bandwidth required for transmission. The input video is converted into frames and each frame is divided into $\mathrm{n} \times \mathrm{n}$ blocks. The blocks are sparsified using OCT as CS can be applied to sparse signals only. The transformed coefficients are ordered in zigzag pattern then reshaped to a sparse vector. The product of measurement matrix and sparse vector is calculated. There are many measurement matrices available like Gaussian matrix, Bernoulli matrix, Toeplitz matrix and Hadamard matrix etc. This prediction based algorithm works well with Gaussian matrix. Many lossless encoding algorithms have been analyzed. The random measurements obtained are quantized and encoded by a CSQPH algorithm for transmission in WSNs.

\section{RESULTS AND ANALYSIS}

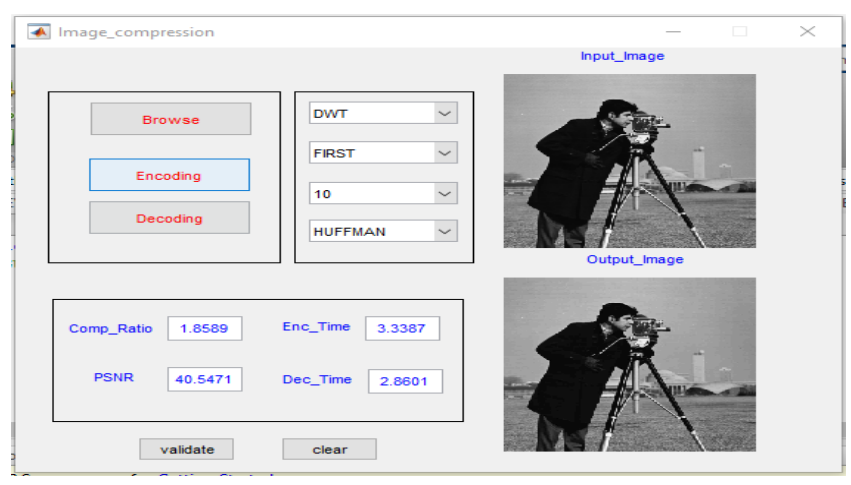

Figure 4: DWT + Huffman

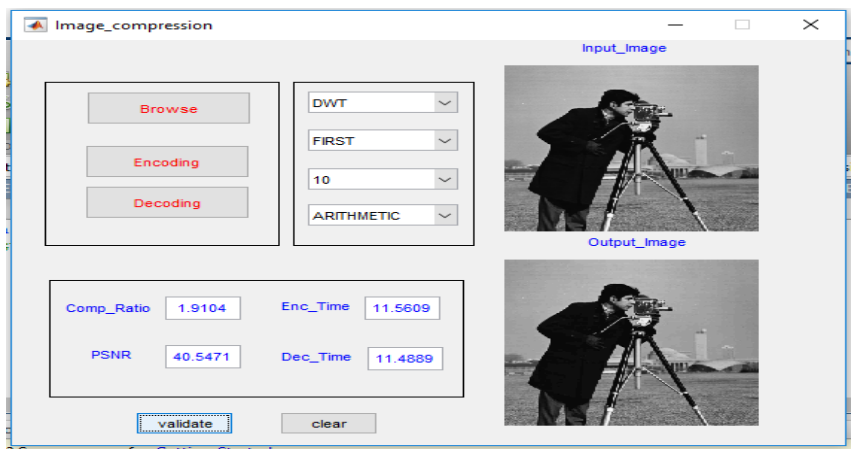

Figure 5: DWT + Arithmetic

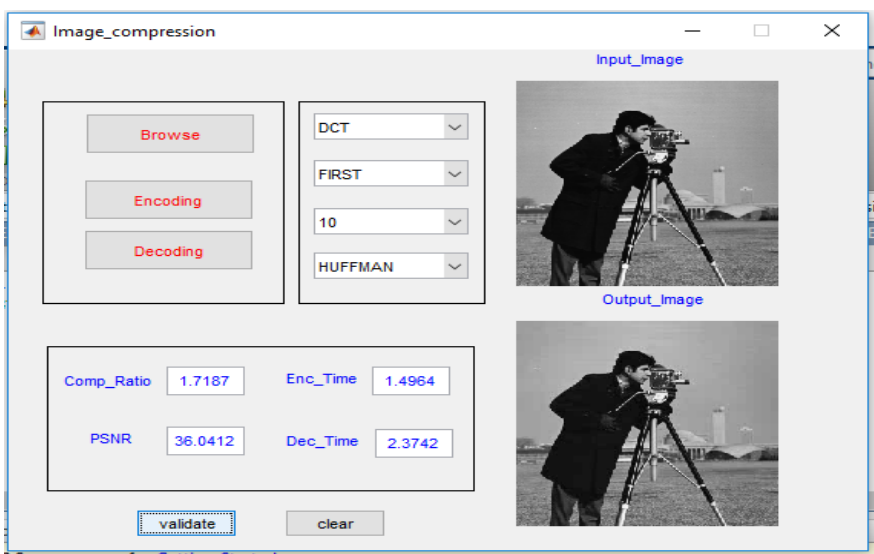

Figure 4: DCT + Huffman

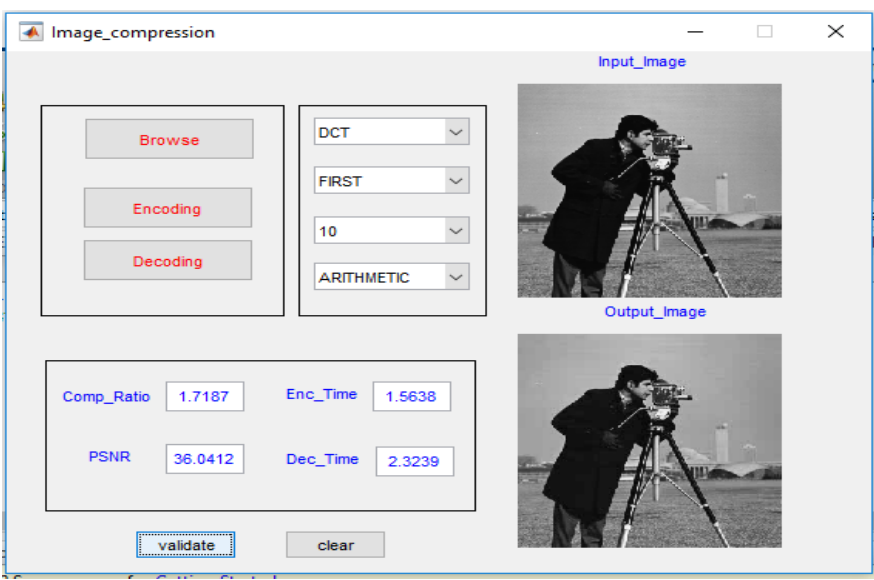

Figure 7: DCT + Arithmetic

TABLE I

ANALYSIS

\begin{tabular}{|c|c|c|c|c|}
\hline Method & $\begin{array}{c}\text { CR } \\
\text { Ratio }\end{array}$ & PSNR & Encoding & Decoding \\
\hline $\begin{array}{l}\text { DWT + } \\
\text { Huffman }\end{array}$ & $\begin{array}{l}1.858 \\
9\end{array}$ & $\begin{array}{l}40.547 \\
1\end{array}$ & 4.8804 & 3.2854 \\
\hline $\begin{array}{l}\text { DWT + } \\
\text { Arithmeti } \\
\text { c }\end{array}$ & $\begin{array}{l}1.910 \\
4\end{array}$ & $\begin{array}{l}40.547 \\
1\end{array}$ & 11.9258 & 11.9751 \\
\hline
\end{tabular}




\begin{tabular}{|l|l|l|l|l|}
\hline $\begin{array}{l}\text { DCT + } \\
\text { Huffman }\end{array}$ & 1.718 & 36.041 & 1.6802 & 2.6985 \\
\hline $\begin{array}{l}\text { DCT + } \\
\text { Arithmeti } \\
\text { c }\end{array}$ & 7.718 & 36.041 & 1.5937 & 2.6409 \\
\hline
\end{tabular}

\section{CONCLUSION}

In this paper, two ways of improving compressive sensing and imaging system's efficiency are demonstrated. Separate methods for subspace sparse sampling is designed to find the superior in the compressive imaging. Compressive imaging and compares two DCT and DWT compressive image conspired real time application and experimental. These methods compressive imaging methods provide predesigned patterns to extract the low frequency formation coupled with random projections broadly used for compressive imaging using different Huffman and arithmetic coding. Results is shows that the DWT with Huffman gives batter PSNR values.

In Future sum up DWT and DCT, gives Hybrid approach will produce large amount of easily achievable image data, so that it would be batter for image processing, transmission and storage abilities.

\section{REFERENCES}

[1] Jian Chenl; Yatin Gao; "Compressive Sensing Image Reconstruction Based on Multiple Regulation Constraints", Springer 2017.

[2] Xiumei Li; Guoan Bi; "Improved Bayesian Compressive Sensing For Image Reconstruction using Single-level Wavelet Transform", IEEE, 2016.

[3] Muhammad Ali Qureshi; M.Deriche; "A new Wavelet based efficient image compression algorithm using compressive sensing", Springer, 2015.

[4] Xiumei Li; Guoan Bi; "Image Reconstruction based on the Improved Compressive Sensing Algorithm”, IEEE 2015.
[5] Narendra N, M Girish Chandra and B S Adiga Innovation Labs, Tata Consultancy Services, Bangalore,India; "Compressive sensing for background subtraction based on Error Correction Coding" ,IEEE, 2015.

[6] Kan Chang; Pak Lun Kevin Ding, and Baoxin Li,Senior Member; "Compressive Sensing Reconstruction of Correlated Imanges using joint Regularization”, IEEE 2015.

[7] Hanxu YOU, Jie ZHU; "Image Reconstruction based on Block-based Compressive Sensing", ACSC, 2015.

[8] Elma Hot,Petar Sekulic; "Compressed Sensing MRI using masked DCT and DFT measurements", MECO,2015.

[9] Sherin C Abraham; Ketki Pathak; Jigna J Patel; "Compressive Sensing Based Image Reconstruction using Wavelet Transform”, IJET, 2017.

[10] Jin ZHANG; Ling XIA; Mei HUANG; Guangrui LI;" Image Reconstruction in Compressed Sensing Based on Single-level DWT", IEEE,2014.

[11] Mohit Kalra; D. Ghosh; "Image Compression Using Wavelet Based Compressed Sensing and Vector Quantization”, IEEE, 2012.

[12] Sonja Grgic, Mislav Grgic, Member, IEEE, and Branka Zovko-Cihlar, Member, IEEE;" Performance Analysis of Image Compression Using Wavelets”, IEEE, 2012.

[13] Simon Foucart; Holger Rahut;"A Mathematical Introduction to Compressive Sensing". 